\title{
GlandVision: A Novel Polar Space Random Field Model for Glandular Biological Structure Detection
}

\author{
$\mathrm{Hao} \mathrm{Fu}{ }^{1}$ \\ http://ima.ac.uk/fu \\ Guoping Qiu ${ }^{1}$ \\ http://www.cs.nott.ac.uk/ qiu/ \\ Muhammad llyas ${ }^{2}$ \\ mohammad.ilyas@nottingham.ac.uk \\ Jie Shu ${ }^{1}$ \\ http://ima.ac.uk/shu
}
${ }^{1}$ School of Computer Science
University of Nottingham
Nottingham, UK
${ }^{2}$ Division of Pathology
School of Molecular Medical Sciences
University of Nottingham
Nottingham, UK

\begin{abstract}
In this paper, we propose a novel method for detecting glandular structures in microscopic images of human tissue. We first transform the image from Cartesian space to polar space and introduce a novel random field model with an efficient inference strategy that uses two simple chain graphs to approximate a circular graph to infer possible boundary of a gland. We then develop a visual feature based support vector regressor (SVR) to verify if the inferred contour corresponds to a true gland. And finally, we combine the outputs of the random field and the regressor to form the GlandVision algorithm for the detection of glandular structures. In the experiments, we treat the task of detecting glandular structures as object (gland) proposal, detection and segmentation problems respectively and show that our new technique outperforms state of the art computer vision algorithms developed for generic objects.
\end{abstract}

\section{Introduction}

Tissue diagnosis is an important part of modern day medicine. Where disease is suspected, tissue samples can be taken from the patient and viewed under the microscope by a Pathologist. In many human tissues, cells are organized into complex anatomical units called glands. In many disease states the glands are disrupted, often in a characteristic fashion. If automated image analysis is to be used to facilitate tissue diagnosis, then recognition of glands is essential.

In this study we sought to devise an algorithm for the automated detection of glandular structures in human tissues. A typical microscopic image of the human colon and the glands contained in it are shown in Fig.1. It can be seen there that a gland is composed of a group of cells who sit side-by-side and form the boundaries. Depending on the way the tissue has been sectioned, the shape of a gland can vary hugely and this poses significant challenge to computational algorithms for automatic gland detection. 


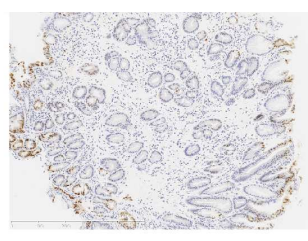

(a)

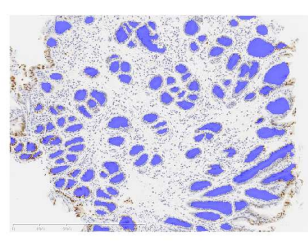

(b)

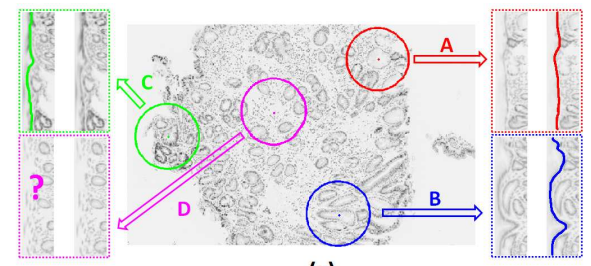

(c)

Figure 1: (a) A microscopic image of human colon tissue containing glands. (b) The glands are manually annotated in blue solid color as shown in the right image. (c) A, B and C correspond to cases where the polar space's origin is inside a gland while D corresponds to the case where the polar space's origin is not inside a gland. We can clearly see a continuous line structure in A, B and C, while this kind of structure can not be seen in D.

In this paper, we proposed a novel method for detecting glandular structures in microscopic images of human tissue. In section 2 we briefly review related literature, and we present our new polar space random field model and a novel efficient inference solution in section 3. In section 4 we introduce a support vector regression model to verify the potential glandular structures. In section 5 we present our overall glandular structure detection method and section 6 presents extensive experimental results and comparison with state of the art.

\section{Related Work}

Since gland exhibits irregular shapes on tissue sections, most of the previous works tackle the gland detection problem by focusing on lumen detection. Lumen is the region in the center of a gland. Previous methods assume that lumen can be identified by their color [四, 四] or texture $[\square, \boldsymbol{\square}]$. Once the lumen area is detected, it is considered as the seed region, and some segmentation methods, such as region growing [ $⿴ 囗 ⿰ ㇇ 丶]$ ], active contour [ $\square]$ ], level-set [ $\square$ ], etc, can be adopted to segment out the glands.

Because color can vary significantly in different microscopic images, these methods sometimes will require human intervention [ $[\nabla]$ to label the lumen regions for model construction. In contrast, our method is totally color-free and processes the grey-scale image. Besides this, we also believe that texture is not an informative cue in this kind of images as can be seen in our experimental section 6.3 where a state-of-art semantic segmentation algorithm which utilizes texture features can only obtain a relatively low performance.

\section{A Novel Random Field Model for Gland Proposal}

One of the most distinctive properties of a gland is that they usually exhibit a closed shape structure. If we place our viewpoint inside the gland, we will see a closed contour, which means if we place the co-ordinate's origin inside a gland and transform the gland to the polar space, we will see a continuous line structure along vertical direction in the polar image. Some examples are shown in Fig.1(c). It is seen that if the origin is inside a gland, we can see an obvious line structure in their corresponding polar image (e.g., regions circled as A, $\mathrm{B}$, and $\mathrm{C}$ ); if the origin is outside a gland there is no such line structure in its polar image 
(e.g., the region circled as D). Based on this observation, the problem of detecting glands can be formulated as the problem of detecting those line structures in the polar image. In this section, we have developed a novel Conditional Random Field (CRF) model to achieve this.

\subsection{A Novel Random Field in the Polar Space}

At each point $\left(x_{0}, y_{0}\right)$ in the Cartesian space, we crop a sub-image with $\left(x_{0}, y_{0}\right)$ at its center. A point $(x, y)$ in the sub-image is transformed to the polar space $(r, \theta)$ using:

$$
\left\{\begin{array}{l}
r=\sqrt{\left(x-x_{0}\right)^{2}+\left(y-y_{0}\right)^{2}} \\
\theta=\arctan \frac{y-y_{0}}{x-x_{0}}
\end{array}\right.
$$

In this work, $\theta$ is discretized to 360 units corresponding to 360 degrees. For a practical system, we only need to consider a limited range of $r \in\left(1, r_{\max }\right)$, which means that we assume the diameter of the maximum size of the gland is $2 * r_{\max }$ (to detect larger glands, we can down-scale the image and still use the same $r_{\max }$ value). After this transformation, a circular region with radius of $r_{\max }$ in the original image is transformed to a fixed size polar image of 360 rows $\times r_{\text {max }}$-columns.

To detect the gland contours in the polar image, we developed a Conditional Random Field (CRF) model [ $[\mathbf{D}]$ ]. A CRF is formally consisted of a random variable $\mathbf{X}$ over the observed data and a set of random variables $\mathbf{Y}=\left\{Y_{1}, Y_{2}, \ldots, Y_{n}\right\}$ over the labels to be inferred. All components $Y_{i}$ of $\mathbf{Y}$ are usually assumed to range over a finite label alphabet $\mathcal{Y}$. In our case, we assign each row of the polar image a label $Y_{i}$, which indicates the position of the gland contour at each row. Accordingly, the label alphabet $\mathcal{Y}$ equals $\left\{1,2, \ldots, r_{\max }\right\}$. The graphical model of our CRF contains only 360 nodes in total and is illustrated in Fig.2(a).

The energy function of our CRF consists of two terms: the unary potential $\psi_{i}$ and the pairwise potential $\psi_{i j}$. It is formally defined as:

$$
E=\sum_{i} \psi_{i}\left(Y_{i} \mid \mathbf{X}\right)+\sum_{i, j} \psi_{i j}\left(Y_{i}, Y_{j} \mid \mathbf{X}\right)
$$

where $\mathbf{X}$ represents the polar image data. It can be represented as $\mathbf{X}=\left\{X_{1}, X_{2}, \ldots, X_{360}\right\}$, where $X_{i}$ corresponds to the $i$ th row in the polar image, as illustrated in Fig.2(b).

We then made two further assumptions about the unary potential and the pairwise potential. For the unary potential $\psi_{i}\left(Y_{i} \mid \mathbf{X}\right)$, we assume it only depends on $X_{i}$ instead of the whole $\mathbf{X}$, and we assume the pairwise potential $\psi_{i j}\left(Y_{i}, Y_{j} \mid \mathbf{X}\right)$ only depends on $X_{i}$ and $X_{j}$. Thus our energy function can be re-written as:

$$
E=\sum_{i} \psi_{i}\left(Y_{i} \mid X_{i}\right)+\sum_{i, j} \psi_{i j}\left(Y_{i}, Y_{j} \mid X_{i}, X_{j}\right)
$$

The factor graph of our CRF is shown in Fig.2(c). The definition of our unary potential and the pairwise potential will be described in section 3.3 and section 3.4.

We also noticed that there exists previous work that also utilize the polar image [ष] or the polar property [四] and adopted a random field model for image segmentation. However, the structure of their random field is quite different from ours. In their work, they assign each pixel a random variable which can take two labels corresponding to 'inside' and 'outside'. For a polar image of 360 rows $\times r_{\max }$-columns, their definition will produce $2^{360 * r_{\max }}=$ $\left(2^{r_{\text {max }}}\right)^{360}$ possible states, whilst our definition will only produce $\left(r_{\max }\right)^{360}$ states, which is much smaller than theirs. Besides, our structure of the random field automatically satisfies the star-convexity property which is considered as an extra constraint in [ $\square]$ ]. 


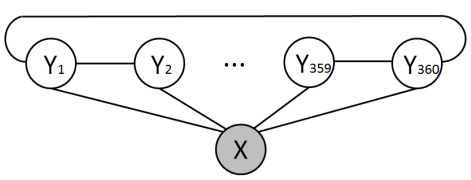

(a)

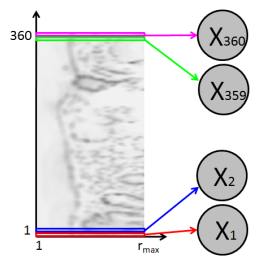

(b)

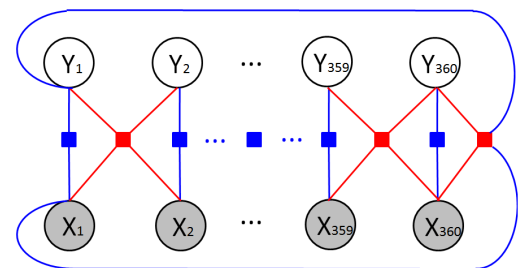

(c)

Figure 2: (a) The graphical model of our CRF model; (b) Each row of the polar image is assigned a random variable; (c) The factor graph of our CRF model.

\subsection{Inference}

The inference procedure of our CRF is to find the optimal $\mathbf{Y}$ that can achieve the lowest energy $E$. From the graph shown in Fig.2(a), we could see that it contains a loop structure, which means we could not perform exact inference, and we had to resort to approximate inference methods, such as loopy belief propagation [ [ ] which is known to be computationally intensive. We noticed that the graph structure in our scenario is a loop structure only if it contains one more edge which links $Y_{1}$ and $Y_{360}$, otherwise it will be a chain, and there exists very efficient inference methods, such as dynamic programming, for chain structure. To avoid the influence of this extra edge, we have developed a novel efficient solution that uses two chain structures to approximate this circulate graph. More specifically, we generate two polar images $I(r, \theta)$, one with a $\theta$ ranges from 0 to $2 \pi$, the other with a $\theta$ ranges from $\pi$ to $3 \pi$. This is shown in Fig.3(a). For these two graphs, we do not connect their heads and tails, hence they are just two chain graphs. We use the well known Viterbi algorithm for inference in this chain structure. The inference is performed separately on each of these two graphs. Finally, we combine the two results together.

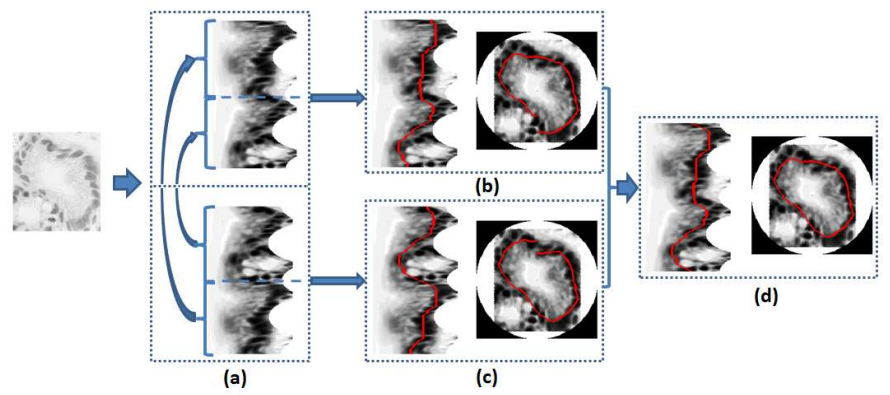

Figure 3: An Efficient Inference Strategy. (a) An image is transformed into two polar images, one's $\theta$ ranges from 0 to $2 \pi$, and the other's ranges from $\pi$ to $3 \pi$. The Viterbi inference algorithm is performed separately on these two polar images, and the results are shown in (b) and (c). These two results are then combined to generate the final result shown in (d).

This 'divide and merge' approach works extremely well in practice, and it only takes a few milliseconds on a $360 \times 100$ pixel polar image. 


\subsection{Unary Potential}

Now we define the unary potential $\psi_{i}\left(Y_{i} \mid X_{i}\right)$ appears in our energy function. We noticed that the gland's boundary usually corresponds to the darker area of the image (see Fig.1 for example). Therefore, a natural strategy is to use the pixel intensity as the unary potential. Let $I\left(r, \theta_{i}\right)$ be the grey value at position $\left(r, \theta_{i}\right)$ in the polar image, then a simple definition of the unary potential can be written as:

$$
\psi_{i}\left(Y_{i}=r\right)=I\left(r, \theta_{i}\right)
$$

As illustrated in Fig.4(c), such simplistic definition is flawed and produces lots of noise. To make the unary potential more informative, we make another stronger assumption that the boundary position prefers a smaller $r$ value. In other words, we prefer the inner contour than the outer contour. This assumption is reasonable because the inner part of a gland is usually in lighter colors. To achieve this, the polar image is first filtered with a vertical edge filter $[-1,0,1]$. Then we calculate the cumulative sum of the edge image in the horizontal direction, and the values in each line of this cumulative edge map are normalized to $(0,1)$. Our new unary potential is defined as:

$$
\psi_{i}\left(Y_{i}=r\right)=1-\left(1-I\left(r, \theta_{i}\right)\right) *\left(1-I_{\text {cumedge }}\left(r, \theta_{i}\right)\right)^{\alpha}
$$

where $\alpha$ is the trade-off parameter between the two terms. Note that only when $I\left(r, \theta_{i}\right)$ and $I_{\text {cumedge }}\left(r, \theta_{i}\right)$ both approaches $0, \psi_{i}\left(Y_{i}=r\right)$ approaches 0 . If either of these two terms approaches $1, \psi_{i}\left(Y_{i}=r\right)$ will approach 1 . We empirically set $\alpha=0.5$ in all our experiments. The complete procedure of generating the unary potential is shown in Fig.4.

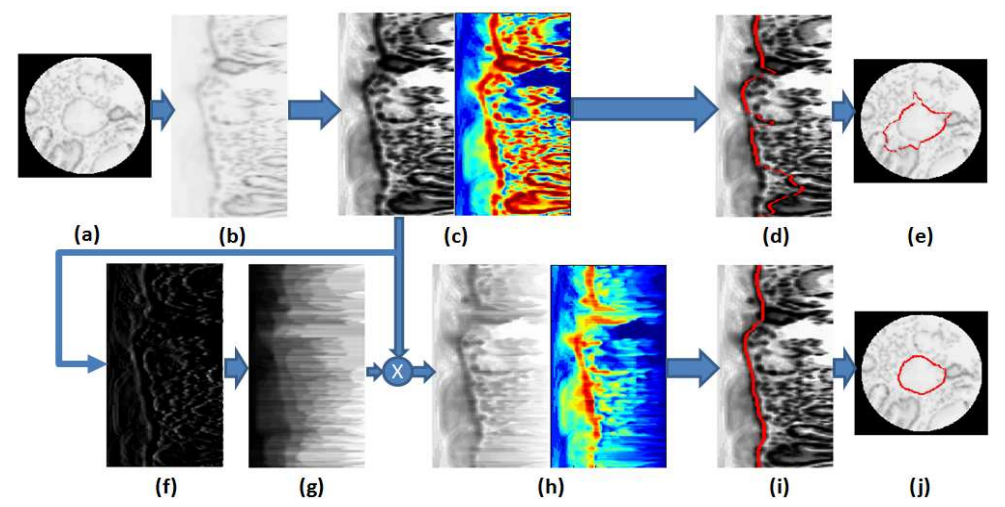

Figure 4: Illustration of Unary Potential. Given an image as shown in (a), it is firstly transformed into the polar space (b). The histogram equalized polar image is shown in (c) left, and the unary potential as defined in (4) is shown in (c) right in pseudo color. This kind of unary potential definition is flawed which will result in bad detection result as shown in (d) and (e). Instead, we first calculate the cumulative edge map (g) based on the edge map (f). Then this cumulative edge map is combined with the original polar image according to (5) to generate our new unary potential shown in (h). Based on this unary potential, we obtain the correct results as shown in (i) and (j). 


\subsection{Pairwise Potential}

As shown in Fig.2(a), edges only exist among neighbouring nodes. In the simplest case, we can adopt the conventional Gaussian edge potential:

$$
\psi_{i j}\left(Y_{i}=r_{i}, Y_{j}=r_{j}\right)=1-\exp \left(-\frac{\left|r_{i}-r_{j}\right|^{2}}{2 \sigma^{2}}\right)
$$

where $\sigma$ is the standard deviation. In practice, we found it is always difficult to set up a suitable $\sigma$ value. That's one of the reasons why we need to adopt a CRF model instead of a Markov Random Field (MRF) model. On defining the pairwise potential, we adopt similar idea from the contrast sensitive Potts model [ㅁ] ]. We draw a line between $\left(r_{i}, \theta_{i}\right)$ and $\left(r_{j}, \theta_{j}\right)$, and we integrate all the intensity values in this line:

$$
I_{\text {sum }}=\int_{\left(r_{i}, \theta_{i}\right)}^{\left(r_{j}, \theta_{j}\right)} I(r, \theta) \mathrm{d}(r, \theta), \quad(r, \theta) \in \operatorname{lin} e_{\left(r_{i}, \theta_{i}\right)}^{\left(r_{j}, \theta_{j}\right)}
$$

Then we calculate the average intensity along this line $I_{a v g}=I_{\text {sum }} /$ length. Our new data dependent pairwise potential is defined as:

$$
\psi_{i j}\left(Y_{i}=r_{i}, Y_{j}=r_{j}\right)=1-\exp \left(-\frac{\left|r_{i}-r_{j}\right|^{2}}{f\left(I_{a v g}\right)}\right)
$$

where $f\left(I_{a v g}\right)$ is a function over $I_{a v g}$. It is formally defined as:

$$
f\left(I_{\text {avg }}\right)=c *\left(\max \left(1-I_{\text {avg }}, \text { thresh }\right)\right)^{\beta}
$$

where $c$, thresh and $\beta$ are all constants. From the above definition of $f\left(I_{a v g}\right)$, we can see that when $I_{a v g}$ approaches $0, f\left(I_{a v g}\right)$ approaches $c$, and when $I_{a v g}$ approaches 1 - thresh, $f\left(I_{a v g}\right)$ approaches $c * t_{\text {thresh }}^{\beta}$. Although we can utilize the training data to learn these parameters, we didn't find the learning procedure much helpful. We empirically set $c=80$, thresh $=0.5$ and $\beta=4$ in all our experiments.

\section{Verification by a Visual Feature based Regressor}

Based on the above gland proposal model, we adopt a sliding window style detection strategy on a given whole image. As our random field model directly searches the radius at each angle, and the radius ranges from 1 to $r_{\text {max }}$, it means our algorithm can detect glands whose radius can vary from 1 to $r_{\max }$. Therefore, we do not need to slide the detecting windows at different scales. Instead, we only need to apply our detection model at each position once. In practice, we sub-sample the pixel locations by 10 pixels, and only apply our model at these positions. In this way, for an image whose size is 1000 pixel width and 1000 pixel height, we will get around 10000 potential gland contours.

The possible gland contours detected by the above random field model still contain much noise, because a true glandular structure is not only defined by its contour but also the structure inside the contour. Therefore, we will design a visual feature based verification module to judge if the contours detected correspond to true glands. We firstly use the energy output of the random field to sort all the potential gland contours. Then a threshold $T$ is set to remove those obvious non-glands. We set $T$ to be a relatively larger value in order to 
maintain a high recall rate. On average, we will retain about 4000 contours among all the 10000 detected contours for each image. For each of these remaining contours, we extract the PHOG visual feature [四] from the smallest bounding box enclosing it. We also compute a score to measures its quality. This score is defined as the maximum overlap between the detected contour $S$ and any ground truth glands $S_{i}$ :

$$
\text { score }=\max _{i} \frac{\left|S \bigcap S_{i}\right|}{\left|S \bigcup S_{i}\right|}
$$

We train a regressor to regress this score using the PHOG features. Recent research have shown that regression is sometimes more suitable than classification for object recognition $[\square, \square]$. We utilize the popular LIBSVM toolbox [0] to learn a nonlinear $\varepsilon-S V R$. The pyramid match kernel defined in [ $[$ ] is used to generate the kernel matrix. In training the nonlinear kernel SVR, we also adopt the 'mining hard negative' strategy [ $\square, \square]$ to incrementally increase the training set. In each step, we only retain the support vectors and add those samples with the largest regression errors.

\section{GlandVision: Integrating Random Field with Regressor}

To perform the final gland detection, we fuse the outputs of the random field and the regressor using a classifier level fusion paradigm []] The random field energy output and the regressor output both have probabilistic meanings. Previous work [ $\square]$ has shown that adding classifier outputs is a more robust way for fusing two probabilistic outputs. We directly sum the two outputs together. Recently, Fu et al. [ $\square$ ] have shown that calibrating the distributions of different measures before combining them can improve performances and we have also tried this approach in the experimental section. The flowchart of our complete gland detection algorithm is depicted in Fig.5.

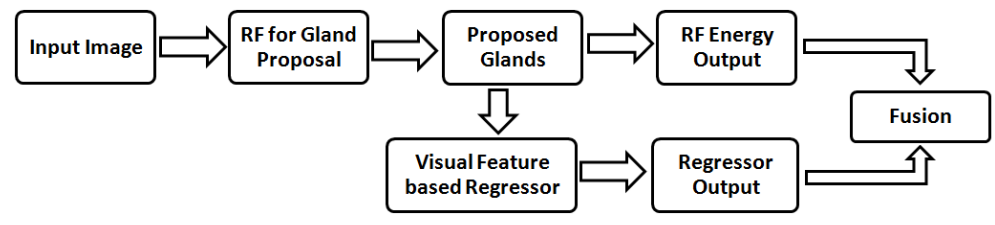

Figure 5: A Complete GlandVision Procedure

\section{Experiments}

We have collected a dataset consisting of 20 high resolution $1280 \times 1024$ pixel microscopic images of human colon tissues. The dataset contains 1072 glands and all have been manually labelled with pixel accuracy, see Fig.1 for a visual example of the images. The dataset and ground truth data will be made public for research purposes in due course. In the experiments, we randomly chose half of these 20 images for training and the rest for testing. As our dataset contains pixel-level ground truth, we can perform different tasks on this dataset. In the following, we will consider three scenarios: Gland Proposal, Gland Detection and Gland Segmentation. 


\subsection{Gland Proposal Accuracy}

It is not until recently that the term of object proposal [山, $\mathrm{Q}, \mathrm{Q}]$ ] have attracted researcher's attention which either advocates the segment-then-recognize strategy [ [] or can facilitate the sliding window object detector [四]. In this theme, our random field model can also be considered as an object (gland) proposal algorithm. In the first experiment, we consider the problem as a gland proposal problem and evaluate the object proposal accuracy of our algorithm against one of the state-of-art algorithms in object proposal [四. Given an image, the task of object proposal is to return a ranked list of bounding boxes that are most likely to contain an object. The performance is evaluated using the Detection-Rate/Signal-To-Noise curve [四]. For the object proposal algorithm in [四], we directly use their public code. The parameters in their method are learned using our training images.

A proposal whose overlap score, as defined in (10), exceeds a predefined threshold is considered as a true positive. A common setting is to set the threshold to be 0.5 , which is the standard PASCAL criterion [ $[\mathrm{\theta}]$. Besides this, we have also considered to set it to 0.8. A higher threshold means that the proposed object and the groundtruth must have a higher degree of overlap hence indicating a higher standard to be considered as true positive.

The results we have achieved together with the objectness method [⿴囗⿴囗十) are shown in Fig.6(a). From there we can see that our method outperforms objectness by a large margin. This comparison is perhaps not fair, as objectness is designed to detect generic objects, while our random field model is specifically designed for gland proposal. We show the result of objectness here to illustrate that gland proposal is a very distinct problem from generic object proposal, and a method that works well for generic object proposal does not necessarily work well in our scenario. Besides this, we could also tell from the figure that our method can achieve a high detection rate (recall rate), even when the overlap threshold is set to 0.8. This indicates our random field model can accurately propose the glands.

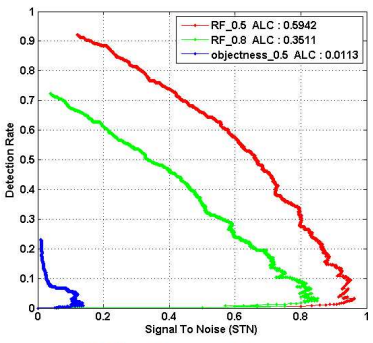

(a) Gland Proposal Accuracy

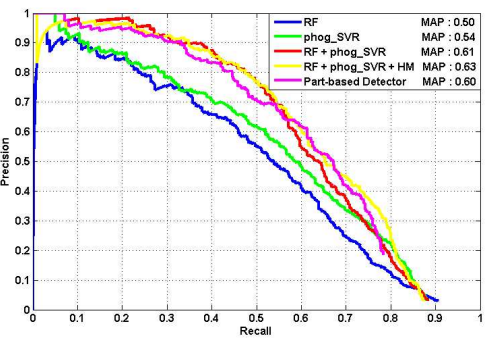

(b) Gland Detection Accuracy

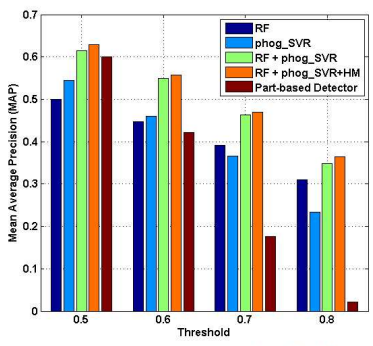

(c) MAP at different thresholds

Figure 6: (a) The DR/STN curve of [⿴囗⿴囗十口] and our Random Field (RF) model at threshold 0.5 (RF_0.5) and 0.8 (RF_0.8). ALC means area to the left of the curve [四]. (b) Precision-Recall curve of different methods in detecting the glands. 'RF' = our Random Field gland proposal model, 'phog_SVR' = our visual feature based regressor, 'RF+phog_SVR' = combining RF and phog_SVR using simple summation, 'RF+phog_SVR + HM' = combining RF and phog_SVR using []. (c) MAP value at different thresholds. 


\subsection{Gland Detection Accuracy}

We now consider the problem as a gland detection problem. For the baseline method, we have implemented the part-based object detection model [ $\square]$ using their public code. This method is considered to be the state of the art method in object detection literature.

For the evaluation criterion, we adopt the Mean Average Precision (MAP) which is the standard PASCAL criterion. Fig.6(b) shows the detection results achieved by our methods together with results of part-based object detection model. On their own, our Random Field and phog_SVR methods achieve a MAP of 0.50 and 0.54 respectively, which is lower than the results obtained by part-based model. However, when we combine these two methods by simply summing their outputs together, our result exceeds part-based model. Besides this, if we adopt the method introduced in [D] to combine the two outputs, our result is further improved. We directly use the histogram matching code by $[\square]$ which is publicly available.

Although our method obtains a higher MAP than the part-based model, the advantage is not obvious. A significant advantage of our model is that it can not only detect the glands, but can also accurately localize them. Therefore, instead of setting the overlap threshold to be 0.5 , we set it to higher values. Note that a higher threshold means higher degree of overlapping between the detected objects and groundtruth hence requiring more accurate localization. We can see from Fig.6(c) that for the part-based detection model, as the threshold increases, MAP drops significantly, whilst for our methods, the drop is not that obvious. When the threshold is set to 0.8, the MAP of part-based detection model drops to 0.02, which means it can hardly detect any glands. But still, our methods can achieve a relatively much higher MAP. Some qualitative examples comparing part-based detection model and our method are shown in Fig.7.

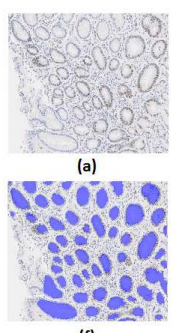

(f)

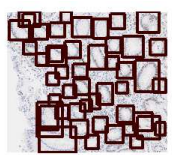

(b)

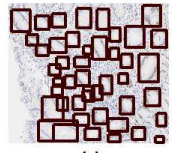

(g)

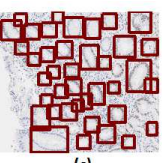

(c)

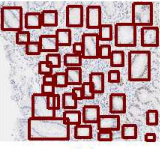

(h)
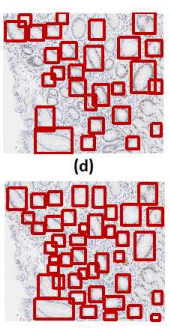

(i)
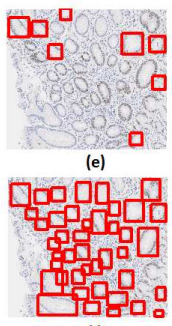

(j)

Figure 7: (a) The original microscopic image. (f) The groundtruth image of glands. (b)(e) The detection results obtained by [ $\square]$ with threshold varies from 0.5 to 0.8 (g)-(j) The results obtained by our model. As the threshold increases, the bounding boxes have to be more accurate. As the accuracy requirements increases (higher threshold), the detection result of [■] dropped significantly.

\subsection{Gland Segmentation Accuracy}

Just as shown in the previous experiments, our model can accurately localize the gland$\mathrm{s}$, which means it can be considered as a segmentation algorithm. In this section, we treat the problem as a semantic segmentation problem, and adopt the PASCAL VOC score $=\frac{T P}{T P+F P+F N}$ as the evaluation criterion. There are in total two semantic classes: 'gland' and 'background'. For the baseline method, we implemented the hierarchical CRF model 
[]] using their public code. This method build a hierarchical graph among pixels and superpixels, and has obtained the best result on some famous semantic segmentation datasets including MSRC21 [D]].

For our segmentation methods, we simply assign all the pixels contained in a gland proposal to be 'gland' class. Here we need to set a hard threshold to judge if a gland proposal is true positive. For the phog_SVR method, we can simply select 0.5 , as this model is trained to have an output ranges from 0 to 1 , but for other models, it is difficult to select an appropriate threshold. Therefore, we count the number of gland proposals whose phog_SVR score is above 0.5 , and chose a threshold that retain the same number of proposals for other methods.

Table 1: Segmentation Accuracy

\begin{tabular}{c|c|c|c|c|c}
\hline & RF & phog_SVR & RF+phog_SVR & RF+phog_SVR+HM & [四] \\
\hline gland & 0.521 & 0.588 & 0.601 & $\mathbf{0 . 6 1 5}$ & 0.119 \\
background & 0.845 & 0.828 & 0.805 & $\mathbf{0 . 8 5 0}$ & 0.785 \\
average & 0.663 & 0.708 & 0.723 & $\mathbf{0 . 7 3 2}$ & 0.452 \\
\hline
\end{tabular}

Table. 1 shows the results obtained by our methods and the method proposed in [ $\mathbb{}$ ]. We can see that [ $[\mathbf{\square}]$ can only obtain an accuracy of 0.119 for the 'gland' class, which means it is very difficult to distinguish the 'gland' class from the 'background' class only based on the features extracted from the pixel or superpixel level. In fact, the inner regions contained in a gland is exactly the same as the regions between glands. It is the circular structure that our model tries to capture that separates the 'gland' class from the 'background' class. An example output of [] and our method is shown in Fig.8.

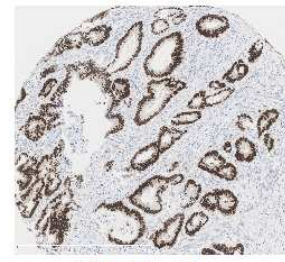

(a) Original Image

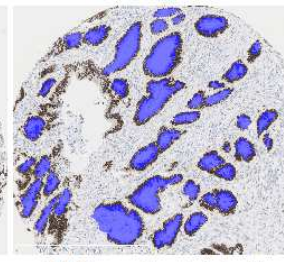

(b) Groundtruth Labeling

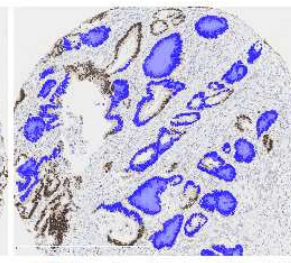

(c) Results obtained by [15]

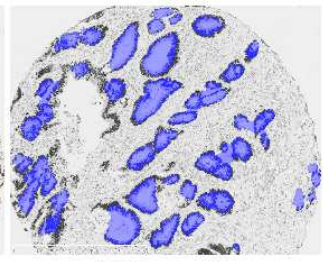

(d) Our results

Figure 8: Segmentation results.

\section{Concluding remarks and future work}

In summary, we have presented a novel method for detecting glandular structures in microscopic images of human colon tissues. We consider the problem at hand from three different angels, i.e. object proposal, object detection and semantic segmentation, and compare our algorithm with several state of the art methods in the respective fields. Different evaluation criteria have confirmed the effectiveness of our methods.

Our method has some limitations. Firstly, due to the utility of the polar image and the structure of the random field model, our method can only detect star-convexity shapes [四]. Secondly, the regressor is trained only based on the PHOG feature, it is anticipated that adding more features could boost the performance of the regressor as well as our final model. 
Thirdly, the glands contained in a microscopic image will never overlap, but we haven't utilized this property in this paper. Future works will try to utilize this property and obtain a global consistent result.

\section{References}

[1] Bogdan Alexe, Thomas Deselaers, and Vittorio Ferrari. WHAT IS AN OBJECT? In CVPR. IEEE, 2010.

[2] Christopher M. Bishop. Pattern Recognition and Machine Learning. 2006.

[3] Matthew B Blaschko and Christoph H Lampert. Learning to Localize Objects with Structured Output Regression. In ECCV, 2008.

[4] A. Bosch, A. Zisserman, and X. Munoz. Representing shape with a spatial pyramid kernel. In Proceedings of the 6th ACM international conference on Image and video retrieval, page 408. ACM, 2007.

[5] Joao Carreira and Cristian Sminchisescu. Constrained Parametric Min-Cuts for Automatic Object Segmentation. In CVPR, number October. IEEE, 2010.

[6] Chih-chung Chang and Chih-jen Lin. LIBSVM : A Library for Support Vector Machines. ACM Transactions on Intelligent Systems and Technology, 2(3):27:1--27:27, 2011.

[7] James Diamond, Neil H. Anderson, Peter H. Bartels, Rodolfo Montironi, and Peter W. Hamilton. The use of morphological characteristics and texture analysis in the identification of tissue composition in prostatic neoplasia. Human Pathology, 35(9):11211131, September 2004. ISSN 00468177.

[8] Ian Endres and Derek Hoiem. Category Independent Object Proposals. In ECCV. Springer, 2010.

[9] Mark Everingham, Luc Gool, Christopher K. I. Williams, John Winn, and Andrew Zisserman. The Pascal Visual Object Classes (VOC) Challenge. International Journal of Computer Vision, 88(2):303-338, September 2009. ISSN 0920-5691.

[10] Reza Farjam, Hamid Soltanian-zadeh, Kourosh Jafari-khouzani, and Reza A Zoroofi. An Image Analysis Approach for Automatic Malignancy Determination of Prostate Pathological Images. Cytometry, 240:227-240, 2007. doi: 10.1002/cyto.b.

[11] Pedro F Felzenszwalb, Ross B Girshick, David McAllester, and Deva Ramanan. Object detection with discriminatively trained part-based models. IEEE transactions on pattern analysis and machine intelligence, 32(9):1627-45, September 2010. ISSN 19393539.

[12] Hao Fu, Guoping Qiu, and Hangen He. Feature Combination beyond Basic Arithmetics. In British Machine Vision Conference(BMVC). BMVA, 2011.

[13] Josef Kirtler, Mohamad Hatefm, Robert P.W.Duin, and Jiri Matas. On combining classifiers. IEEE Trans on Pattern Analysis and Machine Intelligent, 20(3):226-239, 1998. 
[14] Pushmeet Kohli, LâĂŹubor Ladický, and PHS Philip H. S. PHS Torr. Robust Higher Order Potentials for Enforcing Label Consistency. In CVPR, volume 01, 2008.

[15] Lubor Ladicky, Chris Russell, Pushmeet Kohli, Philip H S Torr, and L Ladick. Associative Hierarchical CRFs for Object Class Image Segmentation. In ICCV, pages 739-746. IEEE, September 2009. ISBN 978-1-4244-4420-5.

[16] John Lafferty, Andrew Mccallum, and Fernando Pereira. Conditional Random Fields : Probabilistic Models for Segmenting and Labeling Sequence Data. In ICML, 2001.

[17] Fuxin Li, Joao Carreira, and Cristian Sminchisescu. Object recognition as ranking holistic figure-ground hypotheses. In CVPR. IEEE, 2010.

[18] Ajay Mishra, Yiannis Aloimonos, and Cheong Loong Fah. Active segmentation with fixation. In ICCV, 2009.

[19] Shivang Naik, Scott Doyle, Michael Feldman, John Tomaszewski, and Anant Madabhushi. Gland Segmentation and Computerized Gleason Grading of Prostate Histology by Integrating Low-, High-level and Domain Specific Information. In In Proc. 2nd MICCAI Workshop Microscopic Image Analysis with Appl. in Biology (MIAAB), 2007.

[20] Shivang Naik, Scott Doyle, Michael Feldman, and John Tomaszewski. Automated gland and nuclei segmentation for grading of prostate and breast cancer histopathology. Surgical Pathology, pages 284-287, 2008.

[21] Kien Nguyen, Bikash Sabata, and Anil K. Jain. Prostate cancer grading: Gland segmentation and structural features. Pattern Recognition Letters, 33(7):951-961, May 2012. ISSN 01678655.

[22] Jamie Shotton, John Winn, Carsten Rother, and Antonio Criminisi. TextonBoost: Joint Appearance, Shape and Context Modeling for Multi-Class Object Recognition and Segmentation. In ECCV. Springer, 2006.

[23] Mount Sinai, One Gustave, and L Levy Place. Segmentation of intestinal gland images with iterative region growing. Journal of Microscopy, 220:190-204, 2005.

[24] Olga Veksler. Star Shape Prior for Graph-Cut Image Segmentation. In ECCV, 2008.

[25] Jun Xu, Rachel Sparks, Andrew Janowcyzk, John E Tomaszewski, Michael D Feldman, and Anant Madabhushi. High-Throughput Prostate Cancer Gland Detection, Segmentation, and Classification from Digitized Needle Core Biopsies. Cancer Imaging, pages 77-88, 2010. 